\title{
Development and Research on A Green Scale Inhibitor for Circulating Cooling Water
}

\author{
Xin-ru Guo \\ China Datang Corporation Science \& Technology Research Institute Co., Ltd. Northwest \\ BranchBranch \\ Xi'an 710065 China
}

Key words: green scale inhibitor; circulating cooling water; polyaspartic acid.

\begin{abstract}
In this paper, a kind of green scale inhibitor for circulating cooling water for thermal power plants was studied. The optimum synthesis conditions were determined through experiments. The scale inhibition performance of green scale inhibitors was tested with the actual water of a power plant. Determining the best concentration of scale inhibitor.
\end{abstract}

\section{INTRODUCTION}

Thermal power plants are large industrial water users, of which the thermal power plant cooling water accounts for the vast majority of its water, about $60 \%$ to $70 \%$. Increasing the concentration rate of circulating cooling water can improve the utilization rate of water resources and achieve the purpose of water conservation, which is of great economic and environmental value for thermal power plants that are large water users. Increasing the concentration of circulating water, the key is used for circulating cooling water treatment scale inhibitor, ${ }^{[1}{ }^{1}$ the development of high-performance scale inhibitor has significant economic benefits and important environmental benefits.

At present, thermal power plants have been developed and developed various types of scale inhibitors, commonly used polyphosphate scale inhibitors, organic phosphorus scale inhibitors and polymer scale inhibitors, especially the use of phosphorus scale inhibitor The most extensive, and this scale inhibitor due to phosphorus, can not be naturally degraded, the harm to the environment with a large number of use can not be ignored, with the economic and social development, more and more serious environmental problems on the circulating cooling water agent The requirements are getting higher and higher, a green non-polluting and naturally degradable scale inhibitor becomes an urgent demand for scale inhibitor for circulating water. ${ }^{[2]}$ In this paper, the polyaspartic acid as a green scale inhibitor is studied.

\section{EXPERIMENTAL PART}

\subsection{Reagents and Instruments}

Reagents: Maleic anhydride, ammonia carbonate, calcium chloride, sodium bicarbonate, disodium tetraborate decahydrate, potassium hydroxide, calcium carbonate, ethylenediaminetetraacetic acid (EDTA), hydrochloric acid, sodium hydroxide, methyl red, Calcium red indicator, potassium chloride, are analytical grade.

Instruments: Electronic balance, $\mathrm{pH}$ meter, ultrasonic oscillator, TGA, FTIR, HPLC, NMR, blast drying oven, constant temperature water bath, Gel permeation chromatography (GPC), vacuum oven, centrifuge.

\subsection{Preparation of Polyaspartic Acid}

The preparation of polyaspartic acid mainly consists of two parts: polysuccinimidyl succinate (PSI) made from maleic anhydride; polyaspartic acid (PASP) obtained by saponification of polysuccinimidyl.

\subsubsection{Preparation of Poly Succinimide (PSI)}

The maleic anhydride and ammonia carbonate fixed ratio (molar ratio of 1: 1) was added to the 
beaker, adding an appropriate amount of demineralized water, placed in an oven at $80{ }^{\circ} \mathrm{C}$ reaction $15 \mathrm{~min}$, then the oven temperature was adjusted to $95{ }^{\circ} \mathrm{C}$ constant temperature $10 \mathrm{~min}$, and stirred. And then the temperature was raised to $120{ }^{\circ} \mathrm{C}$ constant temperature $5 \mathrm{~min}$, and finally the temperature was adjusted to $180{ }^{\circ} \mathrm{C} \sim 200{ }^{\circ} \mathrm{C}$ oven thermal polymerization reaction, the time is about $4 \sim 6 \mathrm{~h}$, when the product showed a hard and brittle appearance and foam phenomenon (as shown in Figure 2-1), which means that the intermediate product polysuccinimidate is generated. After the product is washed several times with ethanol, the product on the filter paper is dried in a real oven for 4 hours to obtain the intermediate polysuccinimide.

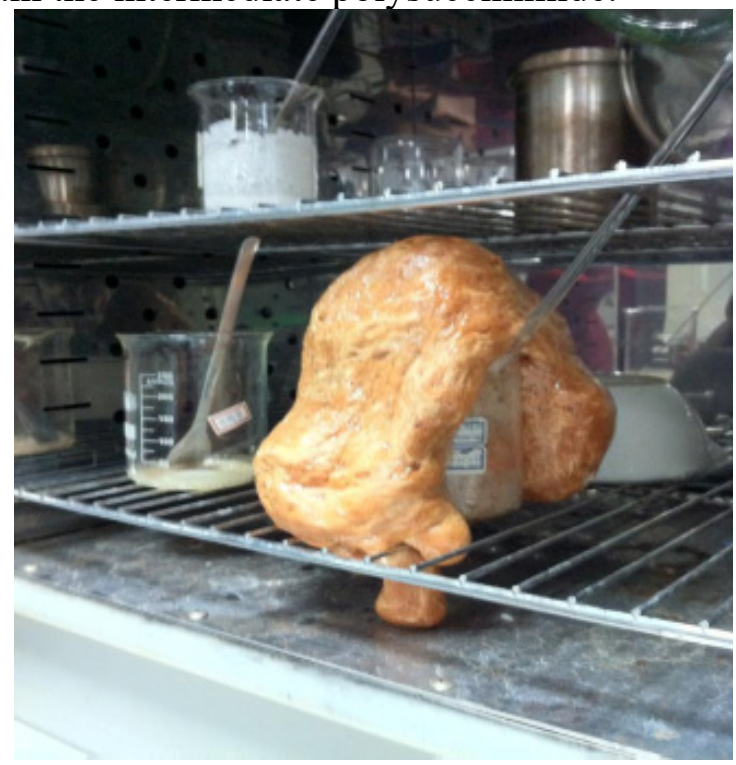

Figure2-1 PSI foaming situation

\subsubsection{Preparation of Polyaspartic Acid (PASP)}

Poly (succinimidyl) polyaspartic acid can be generated by base saponification. $60 \mathrm{~mL}$ of $2 \mathrm{~mol} / \mathrm{L}$ $\mathrm{NaOH}$ aqueous solution was weighed and placed in a $250 \mathrm{~mL}$ flask, and $10 \mathrm{~g}$ of polysuccinic acid imine was added to the sodium hydroxide solution and kept in a thermostatic water bath at $60^{\circ} \mathrm{C}$. for 4 to $6 \mathrm{~h}$. The color of the solution was Dark brown into a light red, as shown in Figure 2-2PSI color change after hydrolysis, before hydrolysis (right); after hydrolysis (left). After the solution is cooled, let stand $12 \mathrm{~h}$, so that the hydrolysis reaction is complete. After the hydrolysis reaction, take a certain amount of $37 \%$ concentrated hydrochloric acid and slowly drip the solution to a $\mathrm{pH}$ value between 7 and 8, and keep stirring for 2 hours to confirm whether the $\mathrm{pH}$ value changes. Take 200

$\mathrm{mL}$ ethanol or methanol slowly dropwise into the hydrolyzed solution, and continue stirring until polyaspartic acid is separated, and then in a vacuum gyrotron concentrator, the ethanol or methanol is completely extracted, while dissolving polyaspartic acid Alkaline aqueous solution were removed together, the concentration bottle placed in a vacuum oven at $100^{\circ} \mathrm{C}$ for 24 hours, and then use a large amount of ethanol or methanol after the synthesis of polyaspartic acid extraction and then placed in a vacuum oven at $100^{\circ} \mathrm{C}$ In dry $4 \mathrm{~h}$, you can get the purified product PASP brown powder, as shown in Figure 2-3 polyaspartic acid solid.

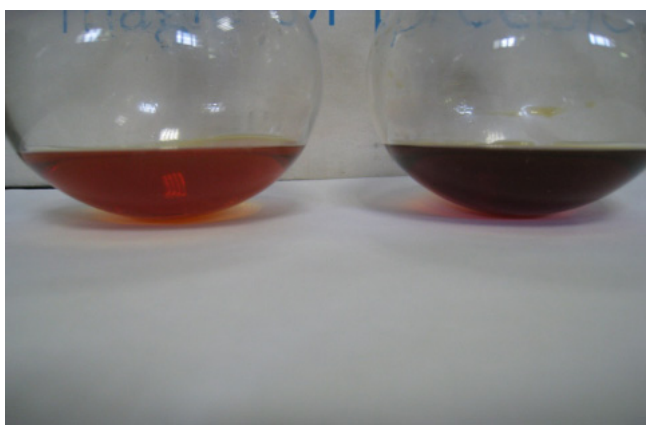

Figure2-2 After PSI hydrolysis color change, before hydrolysis (right); after hydrolysis (left)

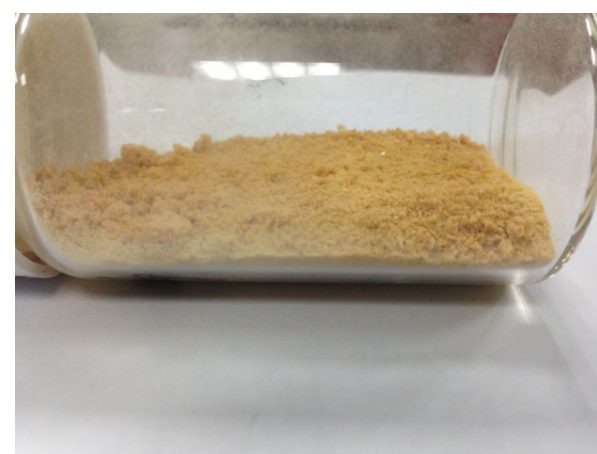

Figure2-3 Poly aspartic acid solid 


\section{SYNTHETIC POLYASPARTIC ACID ANALYSIS}

The synthesized product was analyzed by nuclear magnetic resonance (NMR) and Fourier transform infrared spectroscopy (FTIR), and compared with the standard substance.

\subsection{Nuclear Magnetic Resonance (NMR) Analysis}

Using NMR to analyze the synthesized products, the results are shown in Figure 3-1 to Figure 3-4 for the standard materials.

The comparison between Figure 3-1 and Figure 3-2 shows that the chemical shifts $\delta 2.55, \delta 2.7$ and $\delta 2.8$ belong to the hydrogen atom which is methylene (-CH2-) and belong to Structure of the methine (-CH-) on the hydrogen atoms, $\delta 4.76 \mathrm{D} 2 \mathrm{O}$ structure of the hydrogen atoms.

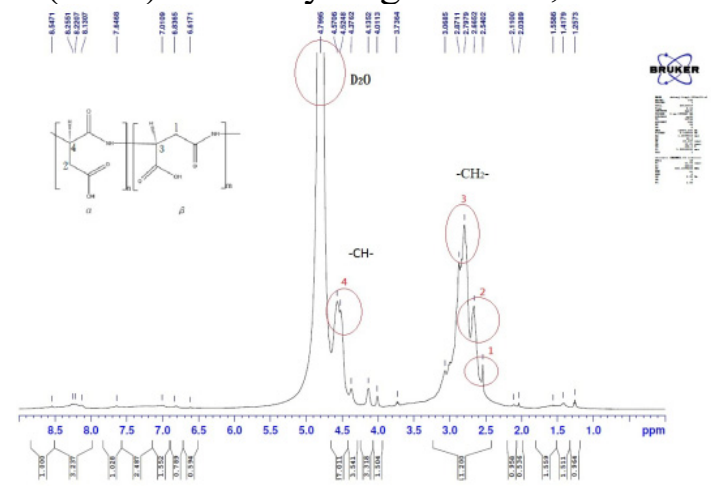

Figure $3-1{ }^{1} \mathrm{H}-\mathrm{NMR}$ Spectrum of Synthesized PASP

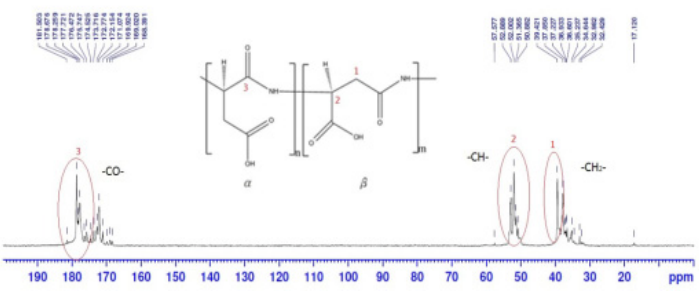

Figure $3-3{ }^{13} \mathrm{C}-\mathrm{NMR}$ carbon spectrum of synthesized PASP

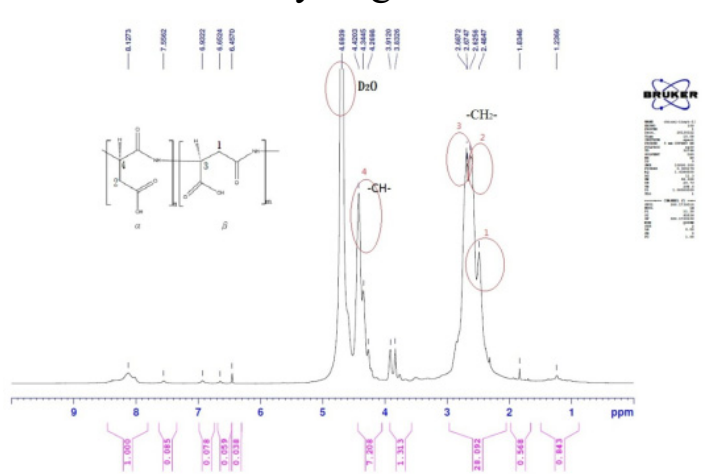

Figure $3-2^{1} \mathrm{H}-\mathrm{NMR}$ Spectrum of Reference Materials

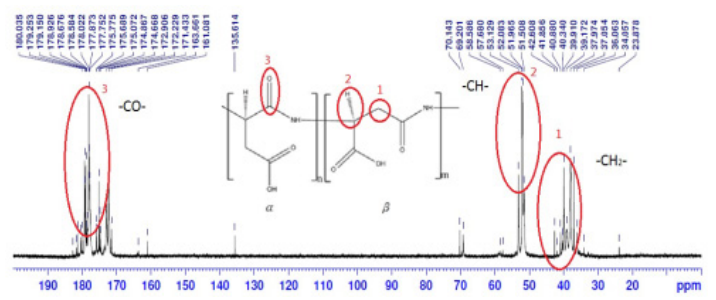

Figure3-41H-NMR spectrum of standard substance

The results of the two samples were analyzed by 13C-NMR as shown in Figure 3-3 and Figure $3-4$, respectively. The results of the comparison showed that: $\delta 175 \sim \delta 182$ on the two carbon spectra belong to the carbonyl group (-CO-) Carbon atoms, $\delta 51-\delta 57$ belong to the carbon atom on the methine group (-CH-), and $\delta 41-\delta 43$ belong to the carbon atom on the methylene group (-CH2-). According to the results of NMR analysis, we can see that the chemical shifts of the synthesized PASP in the NMR spectra are in accordance with the relative chemical shifts of the reference materials, indicating that the structure of the synthesized polyaspartic acid is correct.

\subsection{Fourier Transform Infrared Spectroscopy (FTIR) Analysis}

In order to determine the molecular structure of the synthesized PASP, the standard and the synthetic products were analyzed by Fourier transform infrared spectroscopy. The results are shown in FIGS. 3-5. (A) is a standard substance, and (B) is a synthetic PASP. It can be seen from Fig. 3-5 that the absorption peak between the two functional groups of $\mathrm{NH}-$ and $-\mathrm{OH}$ exists between the wave number $3300-3500 \mathrm{~cm}-1$. Since the NH- bond is stronger than the $-\mathrm{OH}$ bond, the absorption peak of $-\mathrm{OH}$ bond Is covered by the NH-absorption peak in the figure, $1650-1900 \mathrm{~cm}-1$ is the absorption peak of amide bond (peptide bond) -CO-NH-functional group - $\mathrm{CONH}$, and $1600 \mathrm{~cm}-1$ is the absorption peak of COO- functional group, $1400 \mathrm{~cm}-1$ for $-\mathrm{C}=\mathrm{O}$ functional groups of anti-symmetric stretching and symmetric absorption peak, $1300 \mathrm{~cm}-1$ near the functional group $-\mathrm{CN}$ coupling generated absorption peak. It can be confirmed from the spectrum that the compound mainly contains five kinds of functional groups of $\mathrm{NH}-,-\mathrm{C}=\mathrm{O},-\mathrm{CO}-\mathrm{NH}-,-\mathrm{COO}-$, and $\mathrm{CN}$, and the comparison with the reference material can confirm that the poly Aspartic acid structure is correct. 


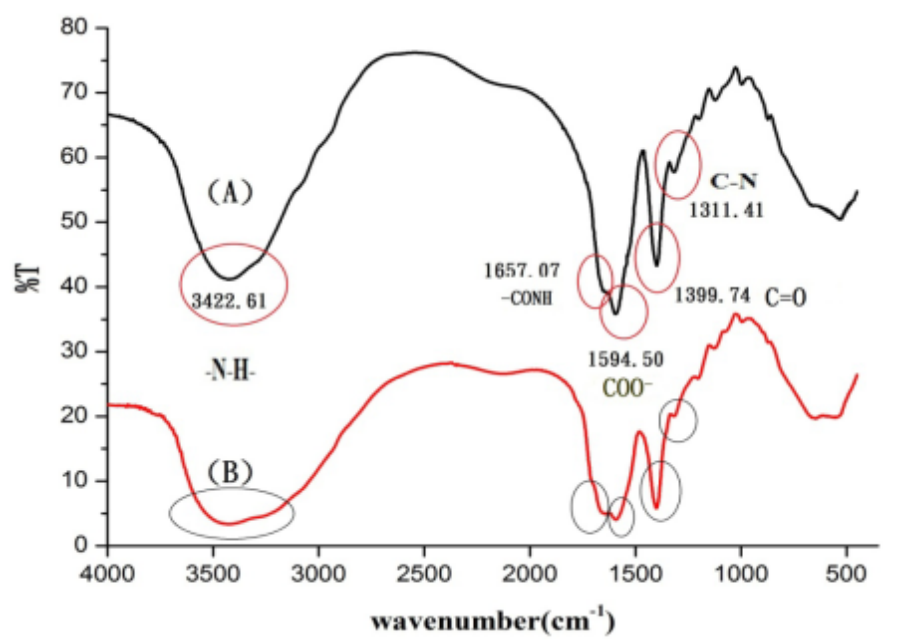

Figure3-5 FTIR profiles of synthetic PASP (B) and standard (A)

\subsection{Summary}

By using nuclear magnetic resonance and Fourier transform infrared spectroscopy analysis shows that the synthesis reaction was successful, the synthesis of polyaspartic acid.

\section{CALCIUM DEPOSITION TEST}

Calcium deposition test is simple, the required equipment and equipment is simple, widely used in the primary election scale inhibitor. Therefore, this part of the calcium deposition test preliminary detection scale inhibition performance of synthetic copolymers, and for the orthogonal test provides a reference test conditions. Calcium deposition tests include calcium carbonate deposition test and calcium phosphate deposition test.

Respectively, configured 8ppm, 10ppm, 12ppm polyaspartic acid solution calcium deposition test, the results shown in Table 4-1.

As can be seen from Table 4-1, polyaspartic acid concentration of 10ppm, calcium carbonate and calcium phosphate, the highest inhibition rate, it can be determined the best dosage of 10ppm.

Table4-1 Calcium deposition test results

\begin{tabular}{cccccccc}
\hline & \multicolumn{2}{c}{ Calcium carbonate deposition test } & \multicolumn{4}{c}{ Calcium phosphate deposition test } \\
$\begin{array}{c}\text { concentrat } \\
\text { ion/ppm }\end{array}$ & $\mathrm{P}_{1}$ & $\mathrm{P}_{2}$ & $\eta_{\mathrm{CaCO} 3}$ & $\mathrm{P}_{0}$ & $\mathrm{P}_{3}$ & $\mathrm{P}_{4}$ & $\eta_{\mathrm{Ca}(\mathrm{PO}) 3}$ \\
& $\mathrm{mg} / \mathrm{ml}$ & $\mathrm{mg} / \mathrm{ml}$ & $/ \%$ & $\mathrm{mg} / \mathrm{ml}$ & $\mathrm{mg} / \mathrm{ml}$ & $\mathrm{mg} / \mathrm{ml}$ & $/ \%$ \\
\hline 8 & & 0.2147 & 86.31 & & & 3.9569 & 78.97 \\
10 & 0.0593 & 0.1763 & 95.68 & 5.0107 & 0 & 4.0421 & 96.67 \\
12 & & 0.1749 & 64.71 & & & 3.7690 & 80.69 \\
\hline
\end{tabular}

\section{CONCLUSION}

The successful synthesis of polyaspartic acid by experiments and the detection of fouling by nuclear magnetic resonance and Fourier transform infrared spectroscopy proved the success of the synthetic experiment. Through calcium deposition test, the optimal dosage of polyaspartic acid was determined to be $10 \mathrm{ppm}$.

\section{REFERENCE}

[1] Akio Yuchi, Yasumasa Gotoh, Shinsuke Itoh, Potentiometry of effective concentration of polyacrylate as scale inhibitor, Analytica Chimica Acta, 2007, 547: 199-203 
[2] Yeung, Dominic W, Terpolymer useful as a scale inhibitor, US, 5282976, 1994

[3] Snyder, William R, Feuerstein, et.al, Method for treating aqueous mediums, US, 4374733, 1983

[4] Kessler S M. Advanced Scale Control Technology for Cooling Water Systems[A]. Annual Conference of National Association of Corrosion Engineerings[C]. Houston, TX, 2002. 02402

[5] Quraishi M A, Singh A, Singh V K. Green approach to corrosion inhibition of mild steel in hydrochloric acid and sulphuric acid solutions by the extract of Murraya koenigii leaves[J]. Materials Chemistry and Physics, 2010, 122(1): 114-122

[6] Ghareba S, Omanovic S. Interaction of 12-aminododecanoic acid with a carbon steel surface: Towards the development of 'green' corrosion inhibitors [J]. Corrosion Science, 2010, 52(6): 2104-2113 